REVIEW ARTICLE

\title{
Analytical Validation of Quantitative Pharmacodynamic Methods used in Clinical Cancer Studies
}

\author{
Dick Pluim $^{1^{*}}$ and Jos H Beijnen ${ }^{1,2}$ \\ ${ }^{1}$ Division of Pharmacology, Netherlands Cancer Institute, Antoni van Leeuwenhoek (NKI-AVL), The Netherlands \\ ${ }^{2}$ Utrecht Institute of Pharmaceutical Sciences (UIPS), Utrecht University, The Netherlands
}

*Corresponding author: Dick Pluim, Division of Pharmacology, Netherlands Cancer Institute, Antoni van Leeuwenhoek (NKI-AVL), Amsterdam, The Netherlands, Tel: +31-620265520

\begin{abstract}
Quantitative pharmacodynamic (PD) methods are used to assess the effect of a drug on its target. The use of these methods in clinical cancer studies has increased enormously with the advent of targeted therapies in the past years. To ensure that quantitative PD methods meet their expectations, methods need to be validated according to internationally recognized standards for amongst others specificity, accuracy, sensitivity, precision and stability. We show that $87 \%$ of our selected clinical cancer studies, published in the last 7 years, used quantitative PD methods that were on average only validated for half of the 10 main validation parameters. Here, we offer our view on the quality of, and the challenges encountered in validation of quantitative PD methods. Finally, we provide future directions and considerations for improvement of the method validation quality.
\end{abstract}

\section{Keywords}

Quantitative, Pharmacodynamic, Biomarker, Validation, Guidelines, Clinical, Cancer

\section{Introduction}

The need for pharmacodynamic (PD) biomarker methods continues to grow with the explosive increase of targeted agents in clinical cancer therapy. PD biomarkers are endogenous biological indicators that can be measured objectively and provide insight about the target engagement, and proof of concept i.e. the extent of the biological effect of a drug. Poor target validation or insufficient target engagement is the main reason for failure of $42 \%$ of clinical phase I and $63 \%$ of phase II clinical cancer trials [1]. Early recognition of these failures is important, which can be accomplished by using PD biomarkers to provide proof of mechanism for the drug-target engagement. PD biomarkers may include molecular, histologic, radiographic, or physiologic characteristics. Most PD biomarkers are, however, molecular biomarkers, which encompass among others protein modifications, enzyme activities, receptor expression and occupancies, and cell counts. In early clinical trials, PD biomarkers can provide useful information for patient management, e.g., whether to continue treatment or to adjust dose (schedule) [2]. They can also help to guide drug dose individualization in targeted/personalized cancer therapy with the aim of treating cancer more effectively and with less toxicity [3]. An illustrative example of the potential usefulness of PD evaluations is the monitoring of B-lymphocyte suppression as PD biomarker in clinical trials to find doses of anti-CD20 monoclonal antibodies required to maximally reduce this cell population. The reduction of the amount of B-cells is presumed to underlie the clinical benefits of these drugs in treating cancer, and has become an established indicator for treatment success [4]. Another example is the monitoring of the inhibition of PARP (target) enzyme activity in white blood cells as PD biomarker for the target engagement of the anti-cancer drug Olaparib [5].

Quantitative PD biomarker methods are used to determine biomarker activity or concentration in different biological matrices such as blood, bile, serum, plasma, urine, tumor cells, and tissue such as skin. For this purpose, these methods need calibration standards prepared in biomarker-free surrogate matrices that are fully defined and representative for the measured biomarkers and their biological matrices. Full analytical

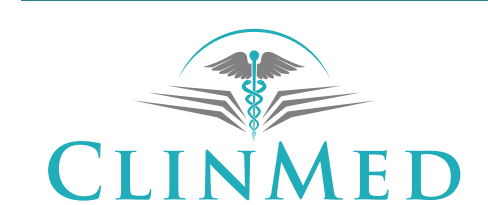

INTERNATIONAL LIBRARY
Citation: Pluim D, Beijnen JH (2021) Analytical Validation of Quantitative Pharmacodynamic Methods used in Clinical Cancer Studies. Int Arch Clin Pharmacol 7:026. doi.org/10.23937/2572-3987.1510026 Accepted: August 18, 2021: Published: August 20, 2021

Copyright: (C) 2021 Pluim D, et al. This is an open-access article distributed under the terms of the Creative Commons Attribution License, which permits unrestricted use, distribution, and reproduction in any medium, provided the original author and source are credited. 
validation of these methods is essential to assure accurate and specific biomarker quantification to prevent interference from other compounds in these matrices. Often biomarkers can fail not because of the underlying science, but because of poor choice of methods and lack of analytical validation [2]. The development and validation of quantitative biomarker methods is, however, more complicated than that of bioanalytical drug methods used for pharmacokinetics due to the following complications. Firstly, sometimes biomarkers need to be detected in more complex biological matrices such as tumor tissues. Secondly, the stability of biomarkers and other endogenous compounds in the biological matrix is often poor. Thirdly, biomarkers are endogenous substances, thus obtaining biomarker-free surrogate matrices for validation of method specificity, and preparation of the calibration curve may be more difficult. Lastly, the biomarker molecule is often not available to act as a certified calibration standard [6].

The last decade has seen significant progress in harmonisation of acceptance criteria, definitions, and guidelines for bioanalytical method validation (BMV) of chromatography and ligand binding methods [7]. For this purpose, the International Council for Harmonisation of Technical Requirements for Pharmaceuticals for Human Use (ICH) recently released BMV Guidance M10 [8]. Fundamentally, ICH-M10 validation involves demonstration of bioanalytical method specificity, accuracy (mean \% deviation from nominal concentration), linearity, dilution integrity, sensitivity, limit of quantification, precision (\% coefficient of variation (CV), and stability for drugs (Table 1). However, international harmonization of guidelines for biomarker method validation is lacking. In their BMV 2018 guideline, the Food and Drug Administration (FDA) recommends to "conduct a full validation of any new bioanalytical method for the analysis of a new drug entity, its metabolite(s), or biomarkers." [9]. The validation of biomarkers thus needs to be consistent with the validation principles applied to bioanalytical drug methods validation [9]. This guideline further states that exploratory quantitative biomarker methods, that are not used to support regulatory decision making, may not require such stringent validation. Exploratory biomarker methods are for instance used to better understand the mechanism of action of drugs, which may ultimately lead to improved future cancer treatments. However, in light of their importance, and to make sure that these methods life up to their expectations, we would argue that also exploratory quantitative biomarker methods, used in clinical trials, need to be fully validated.

Herein, we present our viewpoint on the degree of compliance with the FDA BMV 2018 guideline of quantitative PD biomarker methods used in our selection of clinical cancer trials. Challenges encountered in the process of PD biomarker method development and validation are discussed. The implications of poorly validated methods used for assessment of drug effects are described. In the final section, we offer some considerations and suggestions that may help to improve the quality of PD biomarker methods and consequently their usefulness in clinical cancer trials.

\section{Analytical Methods Validation}

For the assessment of the status of analytical validation of quantitative PD methods used in clinical cancer trials, a focused PubMed literature query was performed on the $8^{\text {th }}$ of December 2020, including the keywords "2013/04/12" [PDAT] : "2020/12/08" [PDAT] AND "humans" [MeSH Terms] AND ("Clinical Trials, Phase I as Topic" [Mesh] OR "Clinical Trials, Phase II as Topic" [Mesh] OR "Clinical Trials, Phase III as Topic"

Table 1: Validation parameters.

\begin{tabular}{|l|l|l|}
\hline \multicolumn{2}{|l|}{ Validation Parameters } & Definition and Criteria \\
\hline I & Specificity & Ability to detect and differentiate biomarker from other (related) substances \\
\hline II & matrix effects & Interfering sample matrix component(s) may not affect accuracy \\
\hline III & Accuracy & Measured and nominal biomarker concentration differ $\leq 15 \%$ \\
\hline IV & Dilution linearity & Extra dilution of quality controls exceeding ULOQ is accurate and precise \\
\hline V & Dilution integrity\# & Normal dilution of samples is accurate and precise \\
\hline VI & Sensitivity/LOD & Lowest biomarker concentration that is significantly above background level \\
\hline VII & LLOQ & Lowest concentration that can be measured with accuracy and precision $\leq 20 \%$ \\
\hline VIII & Precision & Variation in concentration between repeated measurements $\leq 15 \%$ \\
\hline IX & Quality controls & $\begin{array}{l}\text { Prepared in matrix independently from standards; run together with samples to assure validity; } \\
\text { should be within } 20 \% \text { of their nominal concentration }\end{array}$ \\
\hline X & Storage stability & Effect storage conditions on biomarker concentration $\leq 15 \%$ \\
\hline
\end{tabular}

Definition and main criteria of the biomarker validation parameters from the Food and Drug Administration (FDA) Bioanalytical Method Validation (BMV) guideline 2018 [5]. "Because of the narrow range of the calibration standard curve, it is necessary to demonstrate with quality control samples that the analyte of interest, when present in concentrations exceeding the range of quantification (above ULOQ), can be accurately measured by the assay after dilution in blank matrix to bring the biomarker concentrations into the validated range for analysis. An additional reason for conducting dilution linearity experiments is to detect a possible prozone or "hook effect" for ligand binding assays. \#The effect of dilutions used for samples with normal biomarker concentrations on accuracy and precision. LOD = limit of detection; ULOQ and LLOQ = upper and lower limit of quantification. 


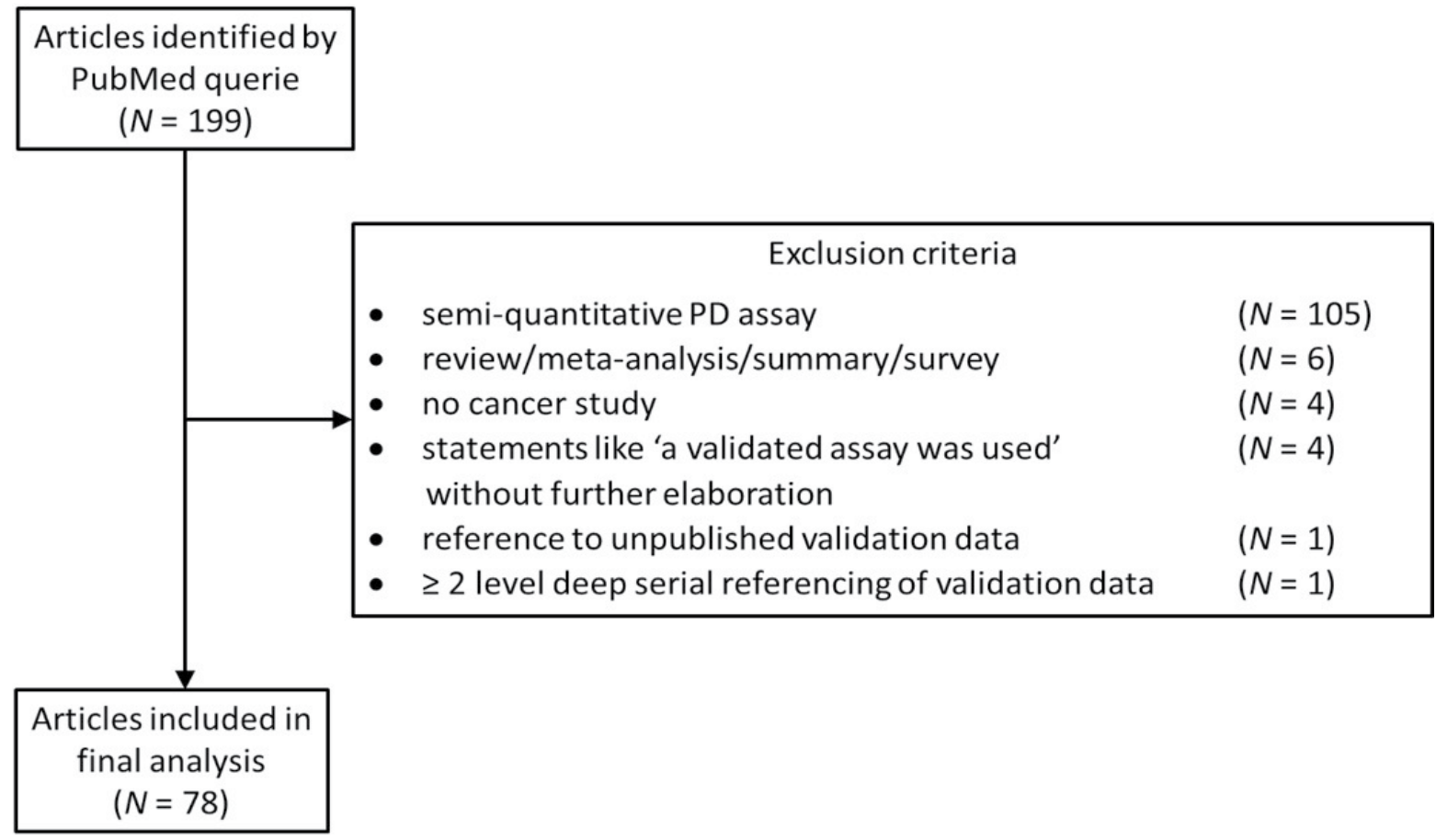

Figure 1: Flow diagram of the published articles selection process.

[Mesh] OR "Clinical Trials, Phase IV as Topic" [Mesh] OR phase I [tiab] OR phase 1 [tiab] OR phase II [tiab] OR phase 2 [tiab] OR phase III [tiab] OR phase 3 [tiab] OR phase IV [tiab] OR phase 4 [tiab] OR "Clinical Trials, Phase I as Topic" [Mesh] OR "Clinical Trials, Phase II as Topic" [Mesh] OR "Clinical Trials, Phase III as Topic" [Mesh] OR "Clinical Trials, Phase IV as Topic" [Mesh]) AND (pharmacodynamic* [tiab] AND method AND methods AND cancer NOT modeling [tiab] NOT review [tiab]). The query returned 199 articles. After review, 4 articles were excluded for failure to provide details about used validation criteria and guidelines. Another article was excluded for referencing to validation data in a submitted, but never published, other article from the authors. Yet another article was excluded for serial referencing. After applying additional exclusion criteria, 78 (39\%) of articles were found suitable for our evaluation using quantitative PD methods in human clinical cancer studies (Figure 1) [10-87]. From each of these articles one quantitative PD method was evaluated and qualitatively scored for compliance with the 10 main validation criteria defined by the FDA BMV 2018 guideline (Table 1 and Table 2).

We found that, 10 PD biomarker methods (13\%) were fully validated in accordance with all 10 main FDA BMV 2018 validation parameters. However, 53 PD methods (68\%) were validated for just on average 5 parameters. Total absence of PD method validation was found for 17 (22\%) of published clinical studies. Compared to chromatographic and ligand binding methods, flow cytometry (FC) methods were relatively less well validated. FC methods were validated for on average just 2 parameters and 50\% lacked any validation. In order to effectively utilize biomarkers to draw meaningful conclusions, it is imperative that the measured concentration or activity of the biomarker in study samples is as close as possible to the actual value at the time of sample collection. The FDA guideline states that investigators should always evaluate their PD methods for stability themselves, referencing the limited sample stability data published in literature is not considered sufficient [9]. For this purpose, stability needs to be assessed for all stocks and reagents used, and for biomarkers during all different parts of the experimental procedure: 1) From sample collection in the clinic to the start of the sample work-up in the lab. 2) On the bench-top or in the autosampler after sample work-up. 3) Long term storage. 4) Freeze-thaw stability. Stability during the sample work-up procedure is by definition not part of the validation, but should be assessed during method development. Nevertheless, only 26 PD methods (33\%) were evaluated for reagent and stock solution stability, and biomarker stability during one or more parts of the experimental procedure.

\section{Challenges and Considerations}

Commercial immuno-assay kits were used in 33 (77\%) of PD methods. These kits are usually developed for general use and therefore may need some level of optimisation, such as adding more calibrators and/or replacing the kit controls in a buffer with controls that mimic the sample matrices. This may prevent accuracy problems from a lack of parallelism between standard curves prepared in buffer and matrix. Many reagents are "research purpose only" (RUO) with considerable batch to batch variation. The use of good manufacturing practice (GMP) reagents or pooled RUO batches should be preferred if possible. A common complaint from 
Table 2: Analytical methods validation.

\begin{tabular}{|c|c|c|c|c|c|c|c|c|c|c|c|c|c|c|c|c|}
\hline \multirow{2}{*}{$\begin{array}{l}\text { Biomarkers } \\
\text { (alphabetically) }\end{array}$} & \multirow[t]{2}{*}{ Assay } & \multirow[t]{2}{*}{ Origin } & \multicolumn{10}{|c|}{ Validation parameters } & \multirow[t]{2}{*}{ Drug } & \multirow[t]{2}{*}{ Phase } & \multirow[t]{2}{*}{ Year } & \multirow[t]{2}{*}{ Ref \# } \\
\hline & & & I & II & III & IV & $\mathbf{V}$ & VI & VII & VIII & IX & $\mathbf{X}$ & & & & \\
\hline ADA & ELISA & LDA & + & + & + & + & + & + & + & $+/+$ & + & + & Blinatumomab & 1 & 2018 & 34 \\
\hline ADA & LBA & Comm. & + & + & + & + & + & - & + & $+/+$ & + & + & Regorafenib & 1 & 2016 & 13 \\
\hline ADA & ELISA & LDA & - & - & - & - & - & + & - & $-/-$ & - & - & TAS-266 & 1 & 2015 & 75 \\
\hline ADA & ELISA & LDA & - & - & - & - & - & + & - & $-/-$ & - & - & Angiocal & 1 & 2013 & 67 \\
\hline Ang2 & ELISA & Comm. & + & + & + & + & + & + & + & $+/+$ & + & + & Docetaxel & 2 & 2014 & 52 \\
\hline B/T-cells & FC & LDA & - & - & - & - & - & - & - & $-/-$ & - & - & Birinapant & 2 & 2016 & 86 \\
\hline BTK & LBA & LDA & + & - & + & + & - & + & + & $+/+$ & - & + & Tirabrutinib & 1 & 2018 & 26 \\
\hline BTK & ELISA & LDA & + & - & - & - & - & + & - & $-/-$ & - & - & Acalabrutinib & 1 & 2018 & 25 \\
\hline ccK18 & ELISA & Comm. & + & + & + & + & + & + & - & $+/+$ & + & + & SOR-C13 & 1 & 2017 & 51 \\
\hline CD137 & xMAP ELISA & Comm. & + & - & + & + & + & + & + & $+/+$ & + & + & Utomilumab & 1 & 2018 & 56 \\
\hline CD26 & FC & LDA & + & - & - & - & - & - & - & $-/-$ & - & - & YS-110 & 1 & 2017 & 58 \\
\hline CD3/14/20 cells & FC & LDA & - & - & - & + & - & - & - & $-/-$ & - & - & CC-223 & 1 & 2015 & 45 \\
\hline CEC & FC & LDA & + & - & + & - & - & - & - & $+/-$ & - & - & Pazopanib & 1 & 2014 & 36 \\
\hline $\mathrm{CgA}$ & RIA & Comm. & - & - & - & - & - & - & + & $+/+$ & - & - & Lanreotide & 3 & 2016 & 31 \\
\hline Collagen IV & ELISA & Comm. & + & - & - & + & - & + & - & $-/-$ & - & - & Cediranib & 1 & 2015 & 35 \\
\hline CRP & LBA & n.d. & - & - & - & - & - & - & + & $-/-$ & - & - & Siltuximab & 1 & 2013 & 21 \\
\hline СТС & $\mathrm{FC}$ & Comm. & + & - & + & + & + & + & + & $+/+$ & + & + & ASP-9521 & $1 / 2$ & 2014 & 85 \\
\hline СТC & FC & Comm. & + & - & + & + & + & + & + & $+/+$ & + & + & ARN-509 & 1 & 2013 & 44 \\
\hline CTC & FC & LDA & - & - & - & - & - & + & - & $-/-$ & - & - & Alisertib & $1 / 2$ & 2017 & 79 \\
\hline СТС & FC & LDA & - & - & - & - & - & - & - & $-/-$ & - & - & TRC105 & 2 & 2016 & 61 \\
\hline mCTC & FC & LDA & + & + & + & + & + & + & + & $+/+$ & + & + & $5-F-2^{\prime}-d C$ & 1 & 2020 & 24 \\
\hline CTX1 & ELISA & Comm. & + & - & - & + & - & + & + & $+/+$ & + & - & GLPG-0187 & 1 & 2016 & 17 \\
\hline Cytokines & xMAP ELISA & Comm. & + & - & + & + & + & + & + & $+/+$ & + & + & TMX-101 & 1 & 2015 & 10 \\
\hline dFdC-TP & LC-MS/MS & LDA & + & - & + & + & + & + & + & $+/+$ & + & + & Gemcitabine & $1 / 2$ & 2015 & 38 \\
\hline DNA & LC-MS/MS & LDA & + & + & + & + & + & + & + & $+/+$ & + & + & LY2603618 & 2 & 2017 & 62 \\
\hline EGF & LBA & LDA & - & - & - & - & - & - & - & $-/-$ & - & - & Sorafenib & 1 & 2015 & 37 \\
\hline EPO & LBA & Comm. & + & + & + & + & - & + & - & $+/+$ & + & - & РТ2385 & 1 & 2018 & 66 \\
\hline FGF & ELISA & Comm. & + & - & - & + & - & - & + & $+/+$ & - & - & S49076 & 1 & 2017 & 70 \\
\hline FGFR & ELISA & LDA & - & - & - & - & - & - & - & $-/-$ & - & - & JNJ-42756493 & 1 & 2015 & 71 \\
\hline G-CSF & ELISA & Comm. & + & + & + & + & + & + & + & $+/+$ & + & - & Lenvatinib & 1 & 2013 & 43 \\
\hline GSH-GSSH & Spectrometry & Comm. & - & - & - & - & - & + & - & $-/-$ & - & - & IACS-010759 & 1 & 2020 & 30 \\
\hline $\mathrm{Y}-\mathrm{H} 2 \mathrm{AX}$ & FC & LDA & - & - & - & - & - & - & - & $-/-$ & - & - & MK-8776 & 1 & 2015 & 42 \\
\hline HSP70 & ELISA & n.d. & - & - & - & - & - & - & - & $-/-$ & - & - & NVP-AUY922 & 2 & 2014 & 77 \\
\hline Y-IF & FC & LDA & - & - & - & - & - & - & - & $-1-$ & - & - & Cixutumumab & 2 & 2014 & 12 \\
\hline IGF1 & ELISA & Comm. & + & - & + & + & - & + & - & $+/+$ & + & - & Polyphenon E & 2 & 2017 & 39 \\
\hline IGF1 & ELISA & Comm. & + & - & + & + & - & - & + & $+/+$ & + & - & MEDI-573 & 1 & 2015 & 54 \\
\hline IGF1R & $\mathrm{FC}$ & LDA & - & - & - & - & - & - & - & $-/-$ & - & - & BIIB-022 & 1 & 2014 & 72 \\
\hline IGFBP2 & ELISA & Comm. & + & + & + & + & + & + & - & $+/+$ & + & - & CNF-1010 & 1 & 2013 & 65 \\
\hline II-2 & ELISA & n.d. & - & - & - & - & - & - & - & $-/-$ & - & - & RO4929097 & 2 & 2015 & 29 \\
\hline II2-10 & ELISA & Comm. & - & - & - & - & - & - & - & $-/-$ & - & - & Ponatinib & 2 & 2019 & 74 \\
\hline II-8 & ELISA & Comm. & + & + & + & + & + & + & + & $+/+$ & + & + & Sunitinib & 3 & 2013 & 87 \\
\hline LFA102 & ELISA & LDA & - & - & - & + & - & - & - & $-/-$ & - & - & LFA-102 & 1 & 2016 & 83 \\
\hline lymphocytes & FC & LDA & - & - & - & - & - & - & - & $-/-$ & - & - & Dinaciclib & 1 & 2013 & 33 \\
\hline monocytes & FC & LDA & - & - & - & - & - & - & - & $-/-$ & - & - & Emactuzumab & 1 & 2019 & 19 \\
\hline p-AKT & ELISA & Comm. & - & + & + & + & + & + & - & $+/+$ & + & - & PX-866 & 2 & 2019 & 82 \\
\hline $\mathrm{p}-\mathrm{AKT}$ & xMAP ELISA & Comm. & + & - & + & + & - & + & - & $+/+$ & - & - & Pictilisib & 1 & 2016 & 41 \\
\hline p-AKT & XMAP ELISA & Comm. & + & - & + & + & + & + & + & $+/+$ & + & + & Pictilisib & 1 & 2015 & 59 \\
\hline
\end{tabular}




\begin{tabular}{|c|c|c|c|c|c|c|c|c|c|c|c|c|c|c|c|c|}
\hline PAR & ELISA & Comm. & + & + & + & + & + & + & + & $+/+$ & + & + & Olaparib & 1 & 2018 & 16 \\
\hline PAR & ELISA & Comm. & + & + & + & + & + & + & + & $+/+$ & + & + & ABT-888 & 1 & 2017 & 57 \\
\hline$p-B A D$ & ELISA & Comm. & + & - & - & + & - & + & - & $+/-$ & - & - & AZD1208 & 1 & 2018 & 46 \\
\hline$p$-CREB & $\mathrm{FC}$ & LDA & + & - & - & - & - & - & - & $+/-$ & - & - & AB-928 & 1 & 2018 & 80 \\
\hline PD1 & ELISA & LDA & + & + & + & + & + & + & + & $+/+$ & + & + & SHR-1210 & 1 & 2018 & 18 \\
\hline PDGF & LBA & Comm. & + & - & - & + & + & - & + & $+/+$ & + & - & Motesanib & 2 & 2015 & 48 \\
\hline PDGF & xMAP ELISA & Comm. & + & - & + & + & + & + & + & $+/+$ & + & + & MEDI-575 & 1 & 2014 & 40 \\
\hline PDGF & ELISA & Comm. & + & + & + & + & + & + & - & $+/+$ & + & - & Pazopanib & 1 & 2013 & 47 \\
\hline PD-L1 & FC & LDA & + & - & - & - & - & - & - & $-/-$ & - & - & Velumab & $1 a$ & 2017 & 53 \\
\hline p-ERK & FC & LDA & - & - & - & - & - & - & - & $-/-$ & - & - & TAK-733 & 1 & 2016 & 73 \\
\hline p-FAK & ELISA & Comm. & + & - & + & + & + & + & - & $+/+$ & + & - & BI 853520 & 1 & 2019 & 60 \\
\hline PI3K & FC & LDA & - & - & - & - & - & - & - & $-/-$ & - & - & Temsirolimus & 1 & 2017 & 78 \\
\hline p-RAD50 & MRM-MS & LDA & + & - & + & + & + & + & + & $+/+$ & + & + & AZD0156 & 1 & 2018 & 14 \\
\hline PRAS40 & ELISA & Comm. & + & - & + & + & + & + & - & $+/+$ & + & - & Azacitidine & 1 & 2017 & 32 \\
\hline p-SCR & ELISA & Comm. & + & - & - & - & - & - & - & $-/-$ & - & - & Dasatinib & 2 & 2019 & 23 \\
\hline Rhodamine & Spectrometry & LDA & + & - & + & - & - & - & - & $+/+$ & - & - & Tariquidar & 1 & 2015 & 68 \\
\hline RON8 & ELISA & Comm. & + & - & - & + & - & - & - & $-/-$ & - & - & Narnatumab & 1 & 2017 & 55 \\
\hline Serum IgG & xMAP ELISA & Comm. & + & - & + & + & + & + & + & $+/+$ & + & + & Sipuleucel-T & 3 & 2015 & 15 \\
\hline serum proteins & xMAP ELISA & Comm. & + & + & + & + & + & + & + & $+/+$ & + & + & Cediranib & 3 & 2014 & 84 \\
\hline s-VEGF & ELISA & Comm. & + & - & + & + & - & + & + & $+/+$ & + & - & Bevacizumab & 2 & 2014 & 81 \\
\hline s-VEGF1/2 & ELISA & n.d. & - & - & - & - & - & - & - & $-/-$ & - & - & Lenvatinib & 1 & 2014 & 69 \\
\hline s-VEGF1/2 & ELISA & Comm. & + & + & + & + & + & + & + & $+/+$ & + & + & Ramucirumab & 3 & 2020 & 22 \\
\hline s-VEGFR2 & ELISA & n.d. & - & - & - & - & - & - & - & $-/-$ & - & - & XL-647 & 1 & 2018 & 49 \\
\hline Testosterone & HPLC & LDA & + & - & + & + & + & + & + & $+/+$ & + & + & Abiraterone & 2 & 2018 & 64 \\
\hline Testosterone & LC-MS/MS & LDA & + & + & + & + & + & + & + & $+/+$ & + & + & Relugolix & 1 & 2015 & 11 \\
\hline Testosterone & LC-MS/MS & LDA & - & - & - & - & - & - & + & $-/-$ & - & - & Orteronel & 1 & 2015 & 27 \\
\hline TSA & radio-assay & LDA & + & + & + & + & + & + & + & $+/+$ & + & + & Capecitabine & 1 & 2020 & 63 \\
\hline VEGF & ELISA & Comm. & + & - & + & + & - & + & + & $+/+$ & + & + & Cediranib & 1 & 2015 & 76 \\
\hline VEGF & ELISA & Comm. & + & - & + & + & - & - & + & $+/+$ & + & - & TRC105 & 1 & 2015 & 20 \\
\hline VEGF-A & LBA & LDA & - & - & - & - & - & - & - & $-/-$ & - & - & Ramucirumab & 1 & 2015 & 50 \\
\hline virus infectivity & Microscopy & LDA & - & - & - & - & - & - & - & $-/-$ & + & - & Enadenotucirev & $1 / 2 a$ & 2019 & 28 \\
\hline
\end{tabular}

Qualitative validation scores for PD assays from 78 phase 1-3 clinical cancer studies. (+) validation in accordance with the Food and Drug Administration bioanalytical method validation guidelines from 2018 [5]; (-) not validated; Ref.\# = article reference number; validation parameters: I = specificity, II = matrix effects, III = accuracy, IV = dilution linearity, $\mathrm{V}=$ dilution integrity, $\mathrm{VI}=$ sensitivity/limit of detection, VII = lower limit of quantification, VIII = within- and between day precision, IX = quality controls, $\mathrm{X}=$ sample storage stability; ADA = anti-drug antibodies; Ang-2 = Angiopoietin-2; BTK = Bruton's Tyrosine Kinase; ccK18 = cleaved cytokeratin 18; $\mathrm{CD}=$ cluster of differentiation; $\mathrm{CgA}=$ chromogranin $\mathrm{A} ; \mathrm{CRP}=\mathrm{C}$-reactive protein; $\mathrm{mCTC}=$ mesenchymal circulating tumor cells; $\mathrm{CEC}=$ circulating endothelial cells; CTX-1 = C-terminal telopeptide of type I collagen; Comm. = commercial assay; EGF = epithelial growth factor; ELISA = enzyme linked immunosorbent sandwich assay; EPO = Erythropoietin; $d F d C-T P=$ gemcitabine triphosphate; FC = flow cytometry; FGFR = fibroblast growth factor receptor; G-CSF = granulocyte colony stimulating factor; GSH-GSSG = glutathione reduced and oxidized; $\mathrm{y}-\mathrm{H} 2 \mathrm{AX}=$ phosphorylated variant histone $2 \mathrm{~A} ; \mathrm{HPLC}=$ high performance liquid chromatography; HSP70 = heat shock protein 70; y-IF = gamma interferon; IGF1R = insulin growth factor receptor; II = interleukin; LFA = Prolactin Receptor Antagonist antibody; LBA = ligand binding assay; LDA = lab developed assay; LC-MS/MS = liquid chromatography tandem mass spectrometry; MRM-MS = multiple reaction monitoring mass spectrometry; n.d. = not disclosed p-AKT = phosphorylated serine/threonine-protein kinase; $P A R=$ poly-adenosine diphosphate ribose; $p$-BAD = phosphorylated B-cell lymphoma antagonist of cell death; $p$-CREB = cAMP Response Element Binding protein; PD1 = programmed death; PDGF $=$ platelet derived growth factor; $P D-L 1=$ programmed death ligand; $p-E R K=$ extracellular-signal-regulated kinase; $p-F A K=F o c a l$ adhesion kinase; PI3K = phosphatidylinositol 3-kinase; -RAD50 = double strand break repair protein; PRAS40 = proline-rich serine/threonine-protein kinase substrate; $p$-SCR = serum creatinine; RON8 = Macrophage-stimulating 1-receptor; VEGFR = vascular endothelial growth factor receptor; TSA = thymidylate synthase activity; RIA = radioimmunoassay; $x M A P=$ multi-analyte profiling.

scientists is that suppliers do not provide the data required to evaluate a given antibody's specificity or its lot-to-lot variability [88]. Companies might ship a batch of antibodies with characterization information derived from a previous batch [88]. And any validation data that are given are often derived under ideal conditions that do not reflect typical experiments [89]. Investigators should be aware that certain suppliers are releasing with 
a fast pace antibodies and ELISA kits of questionable quality [88]. The largest vendors often buy antibodies from smaller suppliers, relabel them and offer them for sale [88]. Such products can lead to unfounded conclusions, waste of many months of research and publications that subsequently need to be retracted. In one illustrative example, the investigators spent 2 years and $\$ 500.000$ because the commercial kit they were using did not recognize Zona Pellucida-like domains protein 1 but Cancer Antigen 125 [90]. Investigators should realise that validation of specificity is the basis of every reliable method. Ideally, researchers would refuse to buy antibodies and immune-assays without extensive validation and quality control data, or would perform the validation themselves. Antibodies should be tested for off-target binding using positive and negative controls e.g. control experiments that involve engineering cell lines to both express and stop expressing the protein of interest. We recommend additional in-house validation before using commercial antibodies and kits, also when obtained from suppliers with a proven track-record, and subsequent entry of the in-house validation data in centralized antibody validation registries such as $\mathrm{CiteAb}$ (www.citeab.com) [91]. CiteAb mines the scientific literature for publications citing the use of individual antibodies, to generate a comprehensive searchable database of literature citations, together with suppliers, blog and news.

Establishing methods "fit for purpose" requires extensive validation and is a continuous process. Scientists, however, often claim that their methods are validated in a very casual way, often without explaining which guidelines were followed or any reference to experimental data to back up their claim. The validation process should start with a description of the purpose of the method, followed by method development and the definition of the performance characteristics. It continues with documentation of the methodology and the validation results. Once the method has been validated, it is crucial that the method is consistently executed as it was validated. The same materials should be used, and system suitability and method controls are applied with the same specifications as during the validation process. During the in-use phase of the methods, there is continuous monitoring to assure that the method still generates results in accordance with the performance characteristics as originally determined. Revalidation is needed if the method is changed, or has been out of use for a while, when it is applied for another material or for a new purpose. For this, the validated method is carefully documented in a procedure or a standard protocol often called a Standard Operating Procedure or 'SOP'. The documentation is not limited to a general description of the methods, but preferably includes all relevant aspects that contribute to the performance of the method.

\section{Future Directions and Outstanding Questions}

Validation of PD biomarker methods is a necessary component for obtaining high-quality PD biomarker data from clinical studies. For this purpose, FDA draft guidelines for the validation of quantitative PD biomarker methods have existed since 2013 [9]. In this light, the incomplete and sometimes total absence of validation for $87 \%$ of quantitative PD biomarker methods in phase 1-3 clinical cancer trials, published in the last 7 years, is quite disturbing. A possible explanation for this lack of validation may be that most quantitative biomarkers used in clinical trials originate from preclinical research in laboratories that are not used to working in accordance with good laboratory practice (GLP). GLP guidelines mandate full validation of quantitative bioanalytical drug methods. For quantitative biomarker methods these guidelines did not exist till the FDA recommended them in their 2013 draft BMV guideline, which became final in 2018 [9].

The FDA BMV 2018 guideline is, however, limited to recommendations for chromatographic and ligand binding method validation [9]. This may explain why we found that validation was most often lacking for quantitative FC biomarker methods [92]. These FC methods are used to quantify cell subpopulations and antigen expression per cell [93]. Quantitative FC methods are used in many clinical biomarker studies, and their importance is increasing [93]. Several factors complicate the validation of these FC methods [94]. One of the most important limitations is lack of standardization in methods, instrument setup, and quality controls. There is an urgent need to address these limitations by establishing internationally harmonized guidelines for quantitative FC biomarker methods used for clinical trials.

Currently, journals apparently do not mandate the use of properly validated quantitative PD methods for clinical studies. Investigators should, however, realize that quantitative PD methods used in clinical trials can only generate reliable and robust results if these methods are fully validated, reliable and robust. In that respect, it does not matter whether these methods are used for regulatory decision making, or for exploratory purposes. Full validation will increase the credibility of PD studies and may increase the discovery rate of qualified clinical PD biomarkers. For this purpose, we recommend that all quantitative PD methods used in clinical trials are fully validated as in the international guidelines for bioanalytical method validation [9].

\section{Acknowledgments}

None

\section{Authors' Contributions}

DP analyzed and interpreted the data. DP and JB 
were major contributors in writing the manuscript. JB supervised the study, provided critical suggestions to the study. All authors read and approved the final manuscript.

\section{Funding}

The authors received no funding for this study.

\section{Availability of Data and Materials}

The datasets used and/or analysed during the current study are available from the corresponding author on reasonable request.

\section{Ethics Approval and Consent to Participate}

Not applicable.

\section{Consent for Publication}

Not applicable.

\section{Competing Interests}

The authors declare that they have no competing interests.

\section{References}

1. Wong $\mathrm{CH}$, Siah KW, Lo AW (2019) Estimation of clinical trial success rates and related parameters. Biostatistics 20: 273-286.

2. Carden CP, Sarker D, Postel-Vinay S, Yap TA, Attard G, et al. (2010) Can molecular biomarker-based patient selection in Phase I trials accelerate anticancer drug development? Drug Discov Today 15: 88-97.

3. Ileana Dumbrava E, Meric-Bernstam F, Yap TA (2018) Challenges with biomarkers in cancer drug discovery and development. Expert Opin Drug Discov 13: 685-690.

4. Gelfand JM, Cree BAC, Hauser SL (2017) Ocrelizumab and other CD20+ B-Cell-depleting therapies in multiple sclerosis. Neurotherapeutics 14: 835-841.

5. de Haan R, Pluim D, van Triest B, van den Heuvel M, Peulen $\mathrm{H}$, et al. (2018) Improved pharmacodynamic (PD) assessment of low dose PARP inhibitor PD activity for radiotherapy and chemotherapy combination trials. Radiother Oncol 126: 443-449.

6. Miller KJ, Bowsher RR, Celniker A, Gibbons J, Gupta S, et al. Workshop on bioanalytical methods validation for macromolecules: Summary report. Pharm Res 18: 13731383.

7. Lowes S, Brown M (2019) Bioanalytical method validation guidance language and a decade of progress. Bioanalysis 11: $587-593$

8. Adoption C, Release C (2019) ICH guideline M10 on bioanalytical method validation 44 .

9. (2018) US Department of Health and Human Services - Food and Drug Administration. Bioanalytical method validation guidance for industry.

10. Arends TJH, Lammers RJM, Falke J, Van Der Heijden AG, Rustighini I, et al. (2015) Pharmacokinetic, pharmacodynamic, and activity evaluation of TMX-101 in a multicenter phase 1 study in patients with papillary nonmuscle-invasive bladder cancer. Clin Genitourin Cancer 13: 204-209

11. MacLean DB, Shi H, Faessel HM, Saad F (2015) Medical castration using the investigational oral $\mathrm{GnRH}$ antagonist TAK-385 (Relugolix): Phase 1 study in healthy males. J Clin Endocrinol Metab 100: 4579-4587.

12. Rajan A, Carter CA, Berman A, Cao L, Ph D, Kelly J, et al. (2014) Cixutumumab - TET p2. Lancet Oncol 15: 191-200.

13. Zopf D, Fichtner I, Bhargava A, Steinke W, Thierauch $\mathrm{KH}$, et al. (2016) Pharmacologic activity and pharmacokinetics of metabolites of regorafenib in preclinical models. Cancer Med 5: 3176-3185.

14. Jones GN, Rooney C, Griffin N, Roudier M, Young LA, et al. (2018) pRAD50: a novel and clinically applicable pharmacodynamic biomarker of both ATM and ATR inhibition identified using mass spectrometry and immunohistochemistry. Br J Cancer 119: 1233-1243.

15. Thakurta DG, Sheikh NA, Fan LQ, Kandadi H, Meagher TC, et al. (2015) Humoral immune response against nontargeted tumor antigens after treatment with sipuleucel-T and its association with improved clinical outcome. Clin Cancer Res 21: 3619-3630.

16. de Haan R, Pluim $D$, van Triest $B$, van den Heuvel $M$, Peulen $H$, et al. (2018) Improved pharmacodynamic (PD) assessment of low dose PARP inhibitor PD activity for radiotherapy and chemotherapy combination trials. Radiother Oncol 126: 443-449.

17. Cirkel GA, Kerklaan BM, Vanhoutte F, Der Aa A Van, Lorenzon G, et al. (2016) A dose escalating phase I study of GLPG0187, a broad spectrum integrin receptor antagonist, in adult patients with progressive high-grade glioma and other advanced solid malignancies. Invest New Drugs 34: 184-192.

18. Mo H, Huang J, Xu J, Chen X, Wu D, et al. (2018) Safety, anti-tumour activity, and pharmacokinetics of fixed-dose SHR-1210, an anti-PD-1 antibody in advanced solid tumours: A dose-escalation, phase 1 study. $\mathrm{Br} \mathrm{J}$ Cancer 119: 538-545.

19. Gomez-Roca CA, Italiano A, Le Tourneau C, Cassier PA, Toulmonde M, et al. (2019) Phase I study of emactuzumab single agent or in combination with paclitaxel in patients with advanced/metastatic solid tumors reveals depletion of immunosuppressive M2-like macrophages. Ann Oncol 30: 1381-1392.

20. Karzai FH, Apolo AB, Cao L, Madan RA, Adelberg DE, et al. (2015) A phase I study of TRC105 anti-endoglin (CD105) antibody in metastatic castration-resistant prostate cancer. BJU Int 116: 546-555.

21. Hudes G, Tagawa ST, Whang YE, Qi M, Qin X, et al. (2013) A phase 1 study of a chimeric monoclonal antibody against interleukin-6, siltuximab, combined with docetaxel in patients with metastatic castration-resistant prostate cancer. Invest New Drugs 31: 669-676.

22. Van Cutsem E, Muro K, Cunningham D, Bodoky G, Sobrero A, et al. (2020) Biomarker analyses of second-line ramucirumab in patients with advanced gastric cancer from RAINBOW, a global, randomized, double-blind, phase 3 study. Eur J Cancer 127: 150-157.

23. Ocana A, Gil-Martin M, Antolín S, Atienza M, Montaño Á, et al. (2019) Efficacy and safety of dasatinib with trastuzumab and paclitaxel in first line HER2-positive metastatic breast cancer: Results from the phase II GEICAM/2010-04 study. Breast Cancer Res Treat 174: 693-701.

24. Coyne GO 'Sulliva, Wang L, Zlott J, Juwara L, Covey JM, et al. (2020) Intravenous 5-fluoro-2'-deoxycytidine administered with tetrahydrouridine increases the proportion 
of p16-expressing circulating tumor cells in patients with advanced solid tumors. Cancer Chemother Pharmacol 85: 979-993.

25. Patel VK, Lamothe B, Ayres ML, Gay J, Cheung JP, et al. (2018) Pharmacodynamics and proteomic analysis of acalabrutinib therapy: Similarity of on-target effects to ibrutinib and rationale for combination therapy. Leukemia 32: $920-930$

26. Yu H, Truong H, Mitchell SA, Liclican A, Gosink JJ, et al. Homogeneous BTK occupancy assay for pharmacodynamic assessment of tirabrutinib (GS-4059/ONO-4059) target engagement. SLAS Discov 23: 919-929.

27. Suzuki K, Ozono S, Yamaguchi A, Koike H, Matsui $H$, et al. (2015) A phase 1 multiple-dose study of orteronel in Japanese patients with castration-resistant prostate cancer. Cancer Chemother Pharmacol 75: 373-380.

28. Machiels JP, Salazar R, Rottey S, Duran I, Dirix L, et al. (2019) A phase 1 dose escalation study of the oncolytic adenovirus enadenotucirev, administered intravenously to patients with epithelial solid tumors (EVOLVE). J Immunother Cancer 7: 4-10.

29. Sylvia M Lee, James Moon, Bruce G Redman, Tarek Chidiac, Lawrence E (2015) A Phase II Study of RO4929097 Gamma-Secretase Inhibitor in Metastatic Melanoma: SWOG 0933. Cancer 121: 432-440.

30. Gammon ST, Pisaneschi F, Bandi ML, Smith MG, Sun Y, et al. (2019) Mechanism-specific pharmacodynamics of a novel complex-I Inhibitor quantified by imaging reversal of consumptive hypoxia with [18F] FAZA PET In Vivo. Cells 8: 1-23.

31. Buil-Bruna N, Dehez M, Manon A, Nguyen TXQ, Trocóniz IF (2016) Establishing the quantitative relationship between lanreotide autogel $\circledast$, chromogranin $A$, and progression-free survival in patients with nonfunctioning gastroenteropancreatic neuroendocrine tumors. AAPS J 18: 703-712.

32. Abaza YM, Kadia TM, Jabbour EJ, Konopleva MY, Borthakur G, et al. (2017) Phase 1 dose escalation multicenter trial of pracinostat alone and in combination with azacitidine in patients with advanced hematologic malignancies. Cancer 123: 4851-4859.

33. Nemunaitis JJ, Small KA, Kirschmeier P, Zhang D, Zhu $Y$, et al. (2013) A first-in-human, phase 1, dose-escalation study of dinaciclib, a novel cyclin-dependent kinase inhibitor, administered weekly in subjects with advanced malignancies. J Transl Med 11: 1.

34. Hijazi Y, Klinger M, Kratzer A, Wu B, Baeuerle PA, et al (2018) Pharmacokinetic and pharmacodynamic relationship of blinatumomab in patients with non-hodgkin lymphoma. Curr Clin Pharmacol 13: 55-64.

35. Gerstner ER, Ye X, Duda DG, Levine MA, Mikkelsen T, et al. (2015) A phase I study of cediranib in combination with cilengitide in patients with recurrent glioblastoma. Neuro Oncol 17: 1386-1392.

36. Hamberg P, Boers-Sonderen MJ, Van Der Graaf WTA, De Bruijn P, Suttle AB, (2014) Pazopanib exposure decreases as a result of an ifosfamide-dependent drug-drug interaction: Results of a phase I study. Br J Cancer 110: 888-893.

37. Do K, Cao L, Kang Z, Turkbey B, Lindenberg ML, et al. (2015) A Phase II study of sorafenib combined with cetuximab in EGFR-expressing, KRAS-mutated metastatic colorectal cancer. Clin Colorectal Cancer 14: 154-161.

38. Leijen S, Burgers SA, Baas P, Pluim D, Tibben M, et al.
(2015) Phase I/II study with ruthenium compound NAMI-A and gemcitabine in patients with non-small cell lung cancer after first line therapy. Invest. New Drugs 33: 201-214.

39. Gee JR, Saltzstein DR, Kim KM, Kolesar J, Huang W, et al. (2017) A phase II randomized, double-blind, presurgical trial of polyphenon e in bladder cancer patients to evaluate pharmacodynamics and bladder tissue biomarkers. Cancer Prev Res 10: 298-307.

40. Becerra CR, Conkling P, Vogelzang N, Wu H, Hong S, et al. (2014) A phase I dose-escalation study of MEDI-575, a PDGFR (alpha) monoclonal antibody, in adults with advanced solid tumors. Cancer Chemother Pharmacol 74: 917-925

41. Ang JE, Pandher R, Ang JC, Asad YJ, Henley AT, et al. (2016) Plasma metabolomic changes following PI3K inhibition as pharmacodynamic biomarkers: Preclinical discovery to phase I trial evaluation. Mol. Cancer Ther. 15: 1412-1424.

42. Daud Al, Ashworth MT, Strosberg J, Goldman JW, Mendelson D, et al. (2015) Phase I dose-escalation trial of checkpoint kinase 1 inhibitor MK-8776 as monotherapy and in combination with gemcitabine in patients with advanced solid tumors. J. Clin. Oncol. 33: 1060-1066.

43. Nishio $M$, Horai $T$, Horiike $A$, Nokihara $H$, Yamamoto $N$, et al. (2013) Phase 1 study of lenvatinib combined with carboplatin and paclitaxel in patients with non-small-cell lung cancer. Br. J. Cancer 109: 538-544.

44. Rathkopf DE, Morris MJ, Fox JJ, Danila DC, Slovin SF, et al. (2013) Phase I study of ARN-509, a novel antiandrogen, in the treatment of castration-resistant prostate cancer. J. Clin. Oncol. 31: 3525-3530.

45. Bendell JC, Kelley RK, Shih KC, Grabowsky JA, Bergsland E, et al. (2015) A phase i dose-escalation study to assess safety, tolerability, pharmacokinetics, and preliminary efficacy of the dual mTORC1/mTORC2 kinase inhibitor CC-223 in patients with advanced solid tumors or multiple myeloma. Cancer 121: 3481-3490.

46. Cortes J, Tamura K, Deangelo DJ, De Bono J, Lorente D, et al. (2018) Phase i studies of AZD1208, a proviral integration Moloney virus kinase inhibitor in solid and hematological cancers. Br. J. Cancer 118: 1425-1433.

47. Bender JLG, Lee A, Reid JM, Baruchel S, Roberts T, et al. (2013) Phase i pharmacokinetic and pharmacodynamic study of pazopanib in children with soft tissue sarcoma and other refractory solid tumors: A children's oncology group phase i consortium report. J. Clin. Oncol. 31: 3034-3043.

48. Bass MB, Yao B, Hei YJ, Ye Y, Davis GJ, et al. (2014) Challenges in developing a validated biomarker for angiogenesis inhibitors: The motesanib experience. PLoS One 9.

49. Das M, Padda SK, Frymoyer A, Molina J, Adjei A, et al. (2018) A safety, tolerability, and pharmacokinetic analysis of two phase I studies of multitargeted small molecule tyrosine kinase inhibitor XL647 with an intermittent and continuous dosing schedule in patients with advanced solid malignancies. Cancer Chemother. Pharmacol. 82: 541550.

50. Chiorean EG, Hurwitz HI, Cohen RB, Schwartz JD, Dalal RP, et al. (2015) Phase I study of every 2- or 3-week dosing of ramucirumab, a human immunoglobulin G1 monoclonal antibody targeting the vascular endothelial growth factor receptor-2 in patients with advanced solid tumors. Ann. Oncol. 26: 1230-1237. 
51. Fu S, Hirte H, Welch S, Ilenchuk TT, Lutes T, et al. (2017) First-in-human phase I study of SOR-C13, a TRPV6 calcium channel inhibitor, in patients with advanced solid tumors. Invest. New Drugs 35: 324-333.

52. Derosa L, Galli L, Orlandi P, Fioravanti A, Di Desidero $\mathrm{T}$, et al. (2014) Docetaxel plus oral metronomic cyclophosphamide: A phase ii study with pharmacodynamic and pharmacogenetic analyses in castration-resistant prostate cancer patients. Cancer 120: 3923-3931.

53. Heery CR, O'Sullivan-Coyne G, Madan RA, Cordes L, Rajan A, et al. (2017) Avelumab for metastatic or locally advanced previously treated solid tumours (JAVELIN Solid Tumor): A phase 1a, multicohort, dose-escalation trial. Lancet Oncol. 18: 587-598.

54. Iguchi $\mathrm{H}$, Nishina $T$, Nogami $N$, Kozuki T, Yamagiwa $\mathrm{Y}$, et al. (2015)Phase i dose-escalation study evaluating safety, tolerability and pharmacokinetics of MEDI-573, a dual IGF-I/II neutralizing antibody, in Japanese patients with advanced solid tumours. Invest. New Drugs 33: 194-200.

55. LoRusso PM, Gounder M, Jalal SI, André V, Kambhampati SRP, et al. (2017) Phase 1 study of narnatumab, an anti-RON receptor monoclonal antibody, in patients with advanced solid tumors. Invest. New Drugs 35: 442-450.

56. Segal NH, He AR, Doi T, Levy R, Bhatia S, et al. (2018) Phase i study of single-agent utomilumab (PF-05082566), a $4-1 \mathrm{bb} / \mathrm{cd} 137$ agonist, in patients with advanced cancer. Clin Cancer Res. 24: 1816-1823.

57. Stoller R, Schmitz JC, Ding F, Puhalla S, Belani CP, et al. (2017) Phase I study of veliparib in combination with gemcitabine. Cancer Chemother. Pharmacol. 80: 631-643.

58. Angevin E, Isambert N, Trillet-Lenoir V, You B, Alexandre $\mathrm{J}$, et al. (2017) First-in-human phase 1 of YS110, a monoclonal antibody directed against CD26 in advanced CD26-expressing cancers. Br. J. Cancer 116: 1126-1134.

59. Sarker D, Ang JE, Baird R, Kristeleit R, Moreno V, et al. (2015) First-in-human Phase I study of Pictilisib ( GDC0941 ), a potent pan-class I phosphatidylinositol-3-kinase ( PI3K ) inhibitor, in patients with advanced solid tumors. Clin Cancer Res. 21: 77-86.

60. de Jonge MJA, Steeghs N, Lolkema MP, Hotte SJ, Hirte HW, et al. (2019) Phase I study of BI 853520, an inhibitor of focal adhesion kinase, in patients with advanced or metastatic nonhematologic malignancies. Target Oncol 14: 43-55.

61. Apolo AB, Karzai FH, Trepel JB, Alarcon S, Lee S, et al. (2017) A phase II clinical trial of TRC105 (Anti-Endoglin Antibody) in adults with advanced/metastatic urothelial carcinoma. Clin Genitourin Cancer 15: 77-85.

62. Laquente B, Lopez-Martin J, Richards D, Illerhaus G, Chang DZ, et al. (2017) A phase II study to evaluate LY2603618 in combination with gemcitabine in pancreatic cancer patients. BMC Cancer 17: 1-9.

63. Roosendaal J, Jacobs BAW, Pluim D, Rosing H, de Vries N, et al. (2020) Phase I pharmacological study of continuous chronomodulated capecitabine treatment. Pharm Res 37: 89.

64. Szmulewitz RZ, Peer C, Ibraheem A, Martinez E, Kozloff $M F$, et al. (2018) Prospective international randomized phase II study of low-dose abiraterone with food versus standard dose abiraterone in castration-resistant prostate cancer. J Clin Oncol 36: 1389-1395.

65. Saif MW, Erlichman C, Dragovich T, Mendelson D, Toft D, et al. (2013) Open-label, dose-escalation, safety, pharmacokinetic, and pharmacodynamic study of intravenously administered CNF1010 (17-(allylamino)-17demethoxygeldanamycin [17-AAG]) in patients with solid tumors. Cancer Chemother Pharmacol 71: 1345-1355.

66. Courtney KD, Infante JR, Lam ET, Figlin RA, Rini BI, et al. (2018) Phase I dose-escalation trial of PT2385, a first-inclass hypoxia-inducible factor-2a antagonist in patients with previously treated advanced clear cell renal cell carcinoma. J Clin Oncol 36: 867-874.

67. Mross K, Richly H, Fischer R, Scharr D, Büchert M, et al. (2013) First-in-human phase i study of PRS-050 (Angiocal), an anticalin targeting and antagonizing VEGF-A, in patients with advanced solid tumors. PLoS One 8: 1-11.

68. Fox E, Widemann BC, Pastakia D, Chen CC, Yang SX, et al. (2015) Pharmacokinetic and pharmacodynamic study of tariquidar (XR9576), a P-glycoprotein inhibitor, in combination with doxorubicin, vinorelbine, or docetaxel in children and adolescents with refractory solid tumors. Cancer Chemother Pharmacol 76: 1273-1283.

69. Koyama N, Saito K, Nishioka Y, Yusa W, Yamamoto N, et al. (2014) Pharmacodynamic change in plasma angiogenic proteins: A dose-escalation phase 1 study of the multikinase inhibitor lenvatinib. BMC Cancer 14: 1-8.

70. Rodon J, Postel-Vinay S, Hollebecque A, Nuciforo P, Azaro A, et al.(2017) First-in-human phase I study of oral S49076, a unique MET/AXL/FGFR inhibitor, in advanced solid tumours. Eur J Cancer 81: 142-150.

71. Tabernero J, Bahleda R, Dienstmann R, Infante JR, Mita A, et al. (2015) Phase I dose-escalation study of JNJ42756493, an oral pan-fibroblast growth factor receptor inhibitor, in patients with advanced solid tumors. J Clin Oncol 33: 3401-3408.

72. Von Mehren M, Britten CD, Pieslor $P$, Saville $W$, Vassos $A$, et al. (2014) A phase 1, open-label, dose-escalation study of BIIB022 (anti-IGF-1R monoclonal antibody) in subjects with relapsed or refractory solid tumors. Invest New Drugs 32: $518-525$

73. Adjei AA, LoRusso P, Ribas A, Sosman JA, Pavlick A, et al. (2017) A phase I dose-escalation study of TAK-733, an investigational oral MEK inhibitor, in patients with advanced solid tumors. Invest New Drugs 35: 47-58.

74. Lee EQ, Muzikansky A, Duda DG, Gaffey S, Dietrich J, et al. (2019) Phase II trial of ponatinib in patients with bevacizumab-refractory glioblastoma. Cancer Med 8: 5988-5994.

75. Papadopoulos KP, Isaacs R, Bilic S, Kentsch K, Huet H, et al. (2015) Unexpected hepatotoxicity in a phase I study of TAS266, a novel tetravalent agonistic Nanobody (®) targeting the DR5 receptor. Cancer Chemother Pharmacol 2015: 887-895.

76. Mark W Kieran, Susan Chi, Stewart Goldman, Arzu OnarThomas, Tina Young Poussaint, et al. (2015) A phase I trial and PK study of cediranib (AZD2171), an orally bioavailable pan-VEGFR inhibitor, in children with recurrent or refractory primary CNS tumors. Childs Nerv Syst 31: 1433-1445.

77. Gaykema SBM, Schröder CP, Vitfell-Rasmussen J, Chua S, Munnink THO, et al. (2014) 89Zr-trastuzumab and 89Zrbevacizumab PET to evaluate the effect of the HSP90 inhibitor NVP-AUY922 in metastatic breast cancer patients. Clin. Cancer Res 20: 3945-3954.

78. Rheingold SR, Tasian SK, Whitlock JA, Teachey DT, Borowitz MJ, et al. (2017) A phase 1 trial of temsirolimus and intensive re-induction chemotherapy for 2 nd or greater 
relapse of acute lymphoblastic leukaemia: A Children's Oncology Group study (ADVL1114). Br J Haematol 177: 467-474.

79. Lin J, Patel SA, Sama AR, Hoffman-Censits JH, Kennedy $B$, et al. (2016) A phase I/II study of the investigational drug alisertib in combination with abiraterone and prednisone for patients with metastatic castration-resistant prostate cancer progressing on abiraterone. Oncologist 21: 1296.

80. Seitz L, Jin L, Leleti M, Ashok D, Jeffrey J, et al. (2019) Safety, tolerability, and pharmacology of AB928, a nove dual adenosine receptor antagonist, in a randomized, phase 1 study in healthy volunteers. Invest New Drugs 37: 711-721.

81. Mahalingam D, Malik L, Beeram M, Rodon J, Sankhala K, et al. (2014) Phase II study evaluating the efficacy, safety, and pharmacodynamic correlative study of dual antiangiogenic inhibition using bevacizumab in combination with sorafenib in patients with advanced malignant melanoma. Cancer Chemother Pharmacol 74: 77-84.

82. Hotte SJ, Chi KN, Joshua AM, Tu D, Macfarlane RJ, et al. (2019) A phase II study of PX-866 in patients with recurrent or metastatic castration-resistant prostate cancer: Canadian cancer trials group study IND205. Clin Genitourin Cancer 17: 201-208.

83. Damiano JS, Rendah KG, Karim C, Embry MG, Ghoddusi $M$, et al. (2013) Neutralization of prolactin receptor function by monoclonal antibody LFA102, a novel potential therapeutic for the treatment of breast cancer. Mol Cancer Ther 12: 295-305.

84. Pommier AJC, Shaw R, Spencer SKM, Morgan SR, Hoff PM, et al. (2014) Serum protein profiling reveals baseline and pharmacodynamic biomarker signatures associated with clinical outcome in $\mathrm{mCRC}$ patients treated with chemotherapy \pm cediranib. Br J Cancer 111: 1590-1604.

85. Loriot Y, Fizazi K, Jones RJ, Van Den Brande J, Molife RL, et al. (2014) Safety, tolerability and anti-tumour activity of the androgen biosynthesis inhibitor ASP9521 in patients with metastatic castration-resistant prostate cancer: Multicentre phase I/II study. Invest New Drugs 32: 995-1004.

86. Anne M Noonan, Kristen P Bunch, Jin-Qiu Chen, Michelle A Herrmann, Jung-min Lee, et al. (2016) Pharmacodynamic markers and clinical results from the Phase II Study of the SMAC-Mimetic birinapant in women with relapsed platinum-resistant or refractory epithelial ovarian cancer. Cancer 122: 588-597.

87. Harmon CS, Deprimo SE, Figlin RA, Hudes GR, Hutson TE, et al. (2014) Circulating proteins as potential biomarkers of sunitinib and interferon-a efficacy in treatment-naive patients with metastatic renal cell carcinoma. Cancer Chemother Pharmacol 73: 151-161.

88. Baker M (2015) Reproducibility crisis: Blame it on the antibodies. Nature 521: 274-276.

89. Taussig MJ, Fonseca C, Trimmer JS (2018) Antibody validation: A view from the mountains. N Biotechnol 45: 1-8.

90. Prassas I, Diamandis EP (2014) Translational researchers beware! Unreliable commercial immunoassays (ELISAs) can jeopardize your research. Clin Chem Lab Med 52: 765766.

91. Helsby MA, Leader PM, Fenn JR, Gulsen T, Bryant C, et al. (2014) CiteAb: A searchable antibody database that ranks antibodies by the number of times they have been cited. BMC Cell Biol 15: 1-12.

92. Davis BH, Wood B, Oldaker T, Barnett D (2013) Validation of cell-based fluorescence assays: Practice guidelines from the ICSH and ICCS - Part I - rationale and aims. Cytom Part B Clin Cytom 84: 282-285.

93. Olga Millán, Mercè Brunet (2015) Flow cytometry as a platform for biomarker discovery and clinical validation. Gen Methods Biomark Res their Appl 1-2: 1-1316.

94. Mizrahi O, Ish Shalom E, Baniyash M, Klieger $Y$ (2018) Quantitative flow cytometry: Concerns and recommendations in clinic and research. Cytom Part B Clin Cytom 94: 211-218. 